EPJ Web of Conferences 37, 06001 (2012)

DOI: $10.1051 /$ epjconf/20123706001

C Owned by the authors, published by EDP Sciences, 2012

\title{
Exclusive meson pair production in proton-proton collisions
}

\author{
Piotr Lebiedowicz ${ }^{1, a}$ and Antoni Szczurek ${ }^{1,2}$ \\ 1 Institute of Nuclear Physics PAN, PL-31-342 Cracow, Poland \\ 2 University of Rzeszów, PL-35-959 Rzeszów, Poland
}

\begin{abstract}
We present a study of the exclusive production of meson pairs in the fourbody $p p \rightarrow p p \pi^{+} \pi^{-}, p p K^{+} K^{-}$reactions at high energies which constitute an irreducible background to resonance states (e.g. $\left.\phi, f_{2}(1270), f_{0}(1500), f_{2}^{\prime}(1525), \chi_{c 0}\right)$. We consider central diffractive contribution mediated by Pomeron and Reggeon exchanges and new diffractive mechanism of emission of pions/kaons from the proton lines. We include absorption effects due to proton-proton interaction and pion/kaon rescattering. Predictions for the total cross section and differential distributions in pion/kaon rapidity and transverse momentum as well as two-pion/kaon invariant mass are presented for the RHIC, Tevatron and LHC colliders. Finally we consider a measurement of exclusive production of a scalar $\chi_{c 0}$ meson via $\chi_{c 0} \rightarrow \pi^{+} \pi^{-}, K^{+} K^{-}$decay.
\end{abstract}

\section{Introduction}

Central exclusive production (CEP) processes of the type $p p \rightarrow p X p$, where $X$ represents the centrally produced state separated from the two very forward protons by large rapidity gaps, significantly extend the physics programme at hadron colliders [1-3]. As discussed in [4,5], the measurement of $\chi_{c 0}$ CEP via two-body decay channels to light mesons is of special interest for both studying the dynamics of heavy quarkonia and for testing the QCD framework of CEP. However, in this case we may expect a sizeable background resulting from meson pair production; such a non-resonant contribution should therefore be carefully evaluated. We have studied the four-body $p p \rightarrow p p \pi^{+} \pi^{-}[6-8], p p K^{+} K^{-}$[9] reactions which constitute an irreducible background to resonance states.

CEP processes have been successfully observed at the Tevatron [3] by selecting events with large rapidity gaps. However, the CDF measurement [10] of $\chi_{c}$ with no other hadrons in the final state, in the $\chi_{c} \rightarrow J / \psi+\gamma$ channel does not allow a separation of the different $\chi_{c J}$ states. It may be possible to isolate the $\chi_{c}$ CEP contribution via two-body decay channels, especially in $\chi_{c J} \rightarrow \pi^{+} \pi^{-}$[4], $K^{+} K^{-}$ [9] and $p \bar{p}$ decay. We recall that such hadronic channels are ideally suited for spin-parity analysis of the $\chi_{c}$ states: in particular the fact that the branching fraction to these channels are relatively larger for scalar meson than for the tensor meson and even absent for the axial meson. A much smaller cross section for $\chi_{c 2}$ production as obtained from theoretical calculation means that only $\chi_{c 0}$ will contribute to the signal $[11,12]$.

A new area of experimental studies of CEP with tagged forward protons and obtained the invariant mass of the pion pair using tracks reconstructed in the STAR Time Projection Chamber (TPC) has just started [2].

At the LHC CEP studies looks also very promising both in the low dipion mass regime and at high masses. In Ref. [13] a possible measurement of the exclusive $\pi^{+} \pi^{-}$production at the LHC with tagged forward protons has been studied. The $p p \rightarrow n n \pi^{+} \pi^{+}$process [14] is also interesting for possible future experiments at high energies.

\footnotetext{
a e-mail: Piotr.Lebiedowicz@ifj.edu.pl
} 


\section{Formalism}
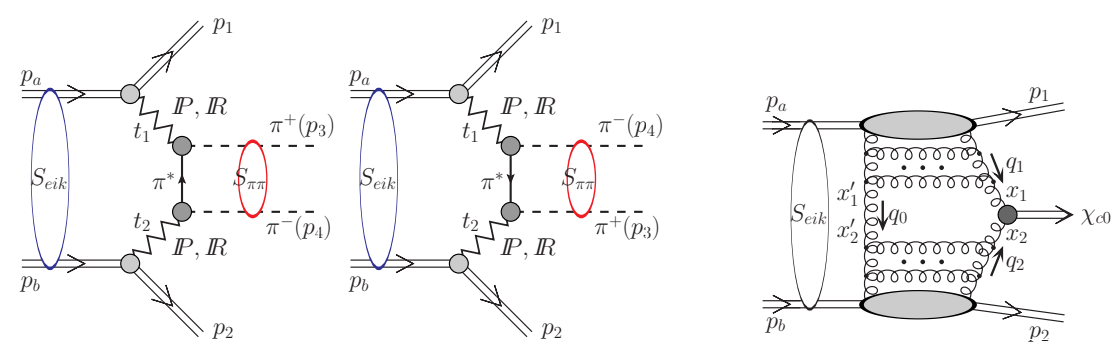

Fig. 1. Left panel: The central diffractive mechanism of exclusive $\pi \pi$ or $K K$ pair production including the absorptive corrections due to proton-proton interactions and pion/kaon rescattering. Right panel: The QCD mechanism of $\chi_{c 0}$ CEP.

The dominant mechanism of the exclusive production of light meson pairs at high energies is sketched in Fig.1 (left panel). The formalism used to calculate of non-resonant background amplitude is explained in detail in Refs [8,9]. The Regge parametrization of the scattering amplitude includes both Pomeron and subleading Reggeon exchanges. Our model with the parameters taken from the Donnachie-Landshoff analysis of the total $\pi N$ or $K N$ cross sections sufficiently well describes the elastic data for energy $\sqrt{s}>3 \mathrm{GeV}$. The form factors correcting for the off-shellness of the intermediate pions/kaons are parametrized as $F_{\pi / K}(\hat{t} / \hat{u})=\exp \left(\frac{\hat{t} / \hat{u}-m_{\pi / K}^{2}}{\Lambda_{o f f}^{2}}\right)$, where the parameter $\Lambda_{o f f}^{2}=2 \mathrm{GeV}^{2}$ is obtained from a fit to the ISR experimental data $[15,16]$.

The QCD amplitude for exclusive central diffractive $\chi_{c 0}$ meson production, sketched in Fig.1 (right panel), was calculated within the $k_{t}$-factorization approach including virtualities of active gluons [11] and the corresponding cross section is calculated with the help of unintegrated gluon distribution functions (UGDFs). In Ref.[4] we have performed detailed studies of several differential distributions of $\chi_{c 0}$ meson production.

\section{Results}

In Fig. 2 we compare our results with CERN ISR experimental data $[15,16]$ at $\sqrt{s}=62 \mathrm{GeV}$. The mass spectra shows strong resonances structure attributed to $f_{0}$ and $f_{2}$ states; their features change with transverse momentum. We see distributions in two-pion invariant mass and in pion rapidity when all (solid line) and only some components in the amplitude are included. Distributions in pion transverse momenta Fig. 3 (upper panel) and in $M_{\pi \pi}$ Fig. 3 (bottom panel) both for the signal $\left(\chi_{c 0}\right)$ and background are presented. The pions from the $\chi_{c 0}$ decay are placed at larger $p_{t, \pi}$. This can be therefore used to improve the signal-to-background ratio by imposing an additional cuts on both pion transverse momenta $\left|p_{t, \pi}\right|>1.5 \mathrm{GeV}$. Measurements of other decay channels, e.g. $K^{+} K^{-}$, are possible as well.

Finally let us discuss results for the $p p \rightarrow p\left(f_{2} \rightarrow \pi^{+} \pi^{-}\right) p$ reaction calculated according to the diagram in Fig.4 (left panel) where the tensorial nature of the Pomeron in the central meson production was used [19]. After the comparison of our results with cross section from existing ISR data we present two-pion invariant mass distribution of continuum where clear signal $f_{2}(1270)$ can be observed. In principle, the resonance and continuum contributions should be added coherently together leading to the distortion of the $f_{2}$ line shape as observe for the $\gamma \gamma \rightarrow f_{2}(1270) \rightarrow \pi^{+} \pi^{-}$reaction [20]. Correlation observables could be particularly sensitive to the spin aspects of the Pomeron. 

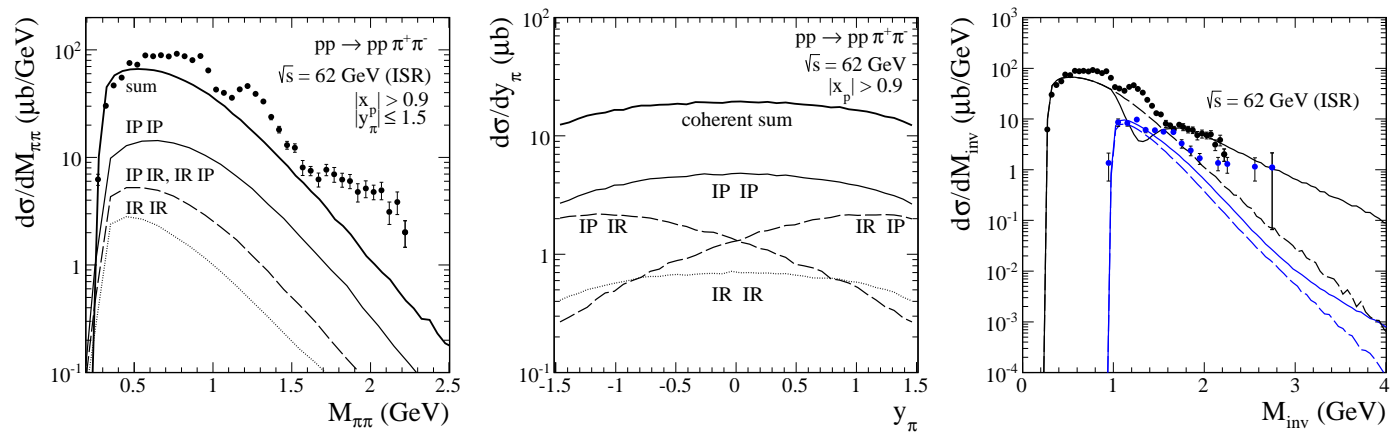

Fig. 2. Left panel: Differential cross section $d \sigma / d M_{\pi \pi}$ at $\sqrt{s}=62 \mathrm{GeV}$ with experimental cuts relevant for the ISR data [16]. The different lines correspond to the situation when all and only some components of the Pomeron and Reggeon exchanges in the amplitude are included (Pomeron-Pomeron component dominates at midrapidities of pions and Pomeron-Reggeon (Reggeon-Pomeron) peaks at backward (forward) pion rapidities, respectively. Central panel: Differential distributions in pion rapidity. The camel-like shape of the distributions is due to the interference of components in the amplitude. Right panel: Comparison of two-pion/kaon invariant mass distribution (black/blue lines, respectively), without (solid lines) and with (dashed lines) rescattering effects in final state. The absorption effects due to $p p$-interaction were included in the calculations.
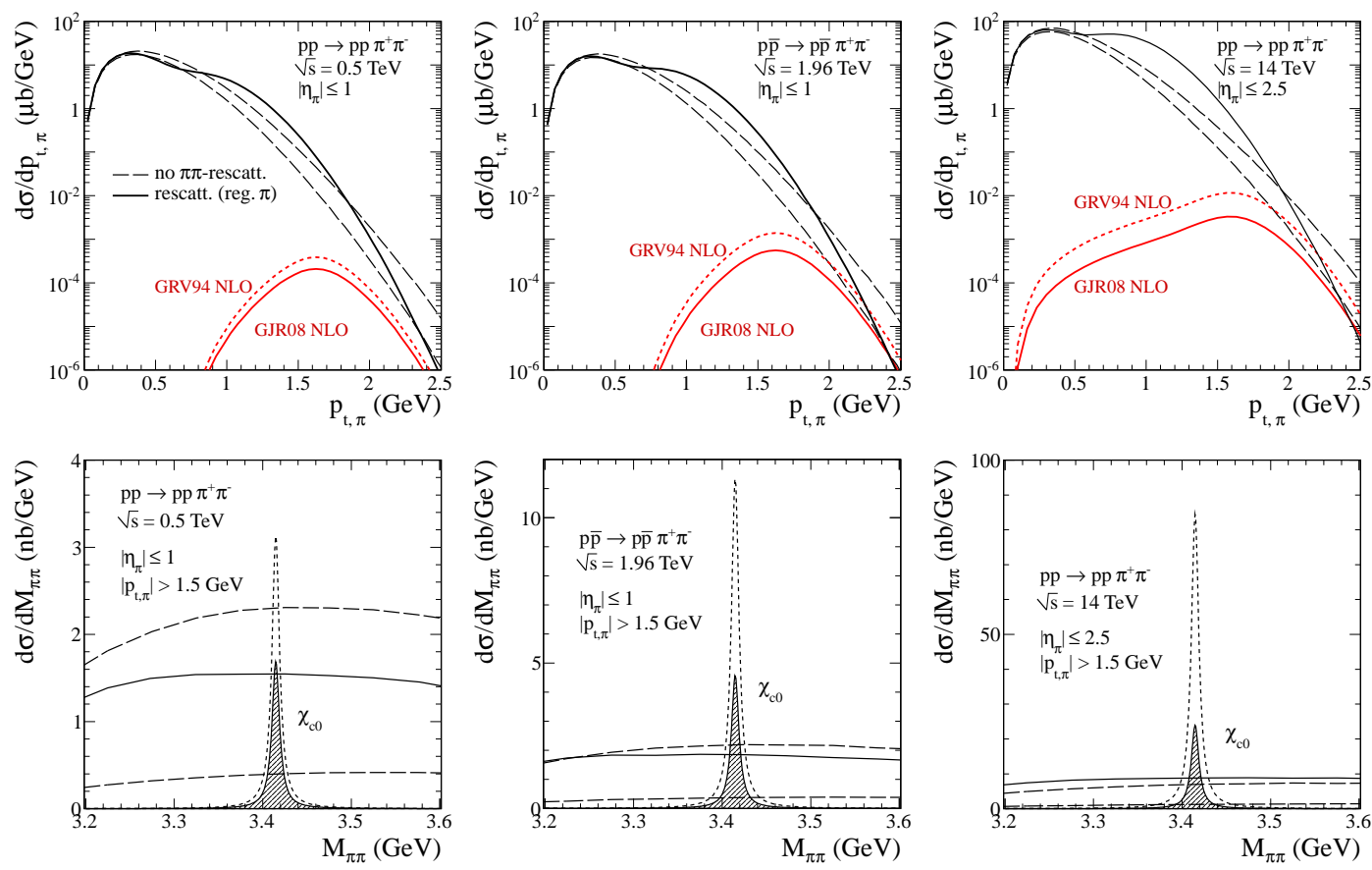

Fig. 3. Upper panel: Differential cross section $d \sigma / d p_{t, \pi}$ at $\sqrt{s}=0.5,1.96,14 \mathrm{TeV}$ with cuts on the pion pseudorapidities. Results for the $\pi \pi$ continuum with the meson propagator and with the cut-off parameter $\Lambda_{o f f}^{2}=1.6$, $2 \mathrm{GeV}^{2}$ (lower and upper dashed lines, respectively) as well as with the generalized pion propagator and $\pi \pi-$ rescattering (solid line) are presented. The absorption effects both for the signal and background were included in the calculations. Bottom panel: The $\pi^{+} \pi^{-}$invariant mass distribution with the relevant restrictions in the pion pseudorapidities and pion transverse momenta. In the calculation of the $\chi_{c 0}$ distributions we have used two choices of collinear gluon distributions: GRV94 NLO (dotted lines) and GJR08 NLO (filled areas). 

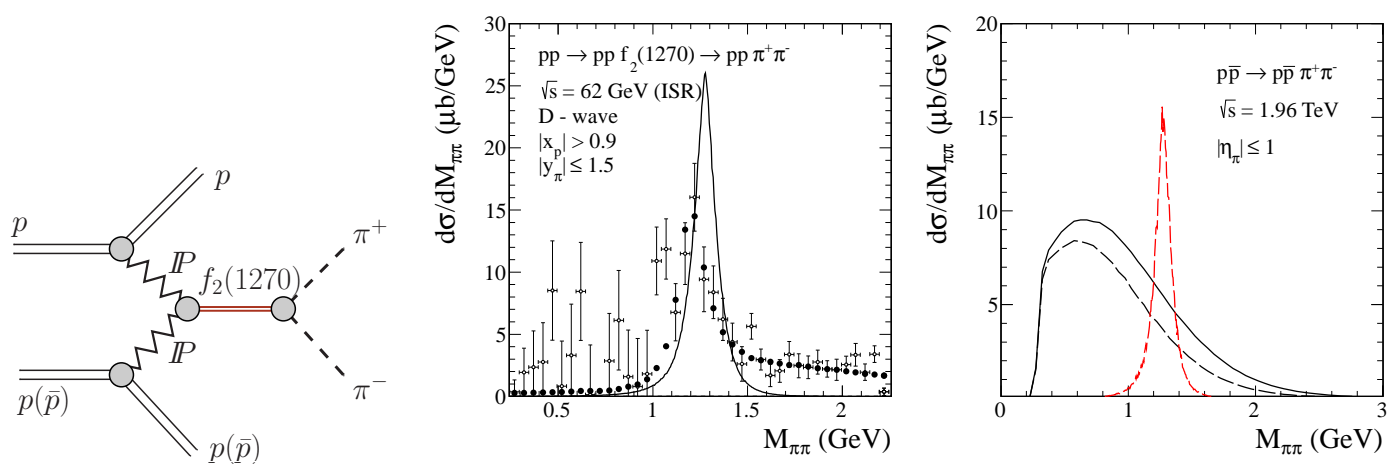

Fig. 4. Left panel: The diffractive mechanism to the central exclusive meson production. Central panel: Comparison of two-pion invariant mass distribution obtained with tensorial Pomeron exchanges with the ISR data [16]. Right panel: Predictions of $d \sigma / d M_{\pi \pi}$ distribution at $\sqrt{s}=1.96 \mathrm{TeV}$.

\section{Conclusions}

We have calculated several differential observables for the $p p \rightarrow p p \pi^{+} \pi^{-}$[6-8] and $p p K^{+} K^{-}$[9] reactions. The full amplitude of central diffractive process was calculated in a simple model with parameters adjusted to low energy data. At high energies the pions or kaons from presented CEP mechanism are emitted preferentially in the same hemispheres, i.e. $y_{3}, y_{4}>0$ or $y_{3}, y_{4}<0$. We have predicted large cross sections for RHIC, Tevatron and LHC which allows to hope that presented by us distributions will be measured in near future. We have calculated also contributions of several diagrams where pions/kaons are emitted from the proton lines. These mechanisms contribute at forward and backward regions and do not disturb the observation of the central DPE component which dominates at midrapidities.

At the Tevatron the measurement of exclusive production of $\chi_{c}$ via decay in the $J / \psi+\gamma$ channel cannot provide production cross sections for different species of $\chi_{c}$. In this decay channel the contributions of $\chi_{c}$ mesons with different spins are similar and experimental resolution is not sufficient to distinguish them. At the LHC situation should be better. We have analyzed a possibility to measure the exclusive production of $\chi_{c 0}$ meson in the proton-(anti)proton collisions at the LHC, Tevatron and RHIC via $\chi_{c 0} \rightarrow \pi \pi, K K$ decay channels. We find that the relative contribution of resonance states and dipion/dikaon continuum strongly depend on the cut on pion/kaon $p_{t}$. The cuts play then a role of the $\pi \pi$ or $K K$ resonance filter. We demonstrated how to impose extra cuts in order to improve the signal-to-background ratio. For a more detailed discussion of this issue see [4,9].

We have found that tensorial Pomeron may equally well describe experimental data on exclusive meson production as the vectorial Pomeron used in the literature. Future experimental data on exclusive meson production at higher energies may give a better answer on the spin structure of the Pomeron and its coupling to the nucleon and mesons. We have shown that relevant measurements at high energies are possible and could provide useful information e.g. about the $f_{0}, f_{2}$ exclusive production.

PL thanks the Organisers for providing excellent scientific environment during the Conference. This work was supported in part by the MNiSW grant No. PRO-2011/01/N/ST2/04116.

\section{References}

1. F. Nerling for the COMPASS Collaboration, in these proceedings; A. Austregesilo and T. Schlueter, arXiv:1207.0949.

2. J. Turnau for the STAR Collaboration, in these proceedings.

3. M. Albrow, A. Swiech and M. Zurek, in these proceedings.

4. P. Lebiedowicz, R. Pasechnik and A. Szczurek, Phys. Lett. B701 (2011) 434. 
5. L.A. Harland-Lang, V.A. Khoze, M.G. Ryskin and W.J. Stirling, arXiv:1204.4803; arXiv:1105.1626.

6. A. Szczurek and P. Lebiedowicz, Nucl. Phys. A826 (2009) 101.

7. P. Lebiedowicz, A. Szczurek and R. Kamiński, Phys. Lett. B680 (2009) 459.

8. P. Lebiedowicz and A. Szczurek, Phys. Rev. D81 (2010) 036003.

9. P. Lebiedowicz and A. Szczurek, Phys. Rev. D85 (2012) 014026.

10. T. Aaltonen et al. [CDF Collaboration], Phys. Rev. Lett. 102 (2009) 242001.

11. R.S. Pasechnik, A. Szczurek and O.V. Teryaev, Phys. Rev. D78 (2008) 014007; Phys. Lett. B680 (2009) 62; Phys. Rev. D81 (2010) 034024.

12. L.A. Harland-Lang, V.A. Khoze, M.G. Ryskin and W.J. Stirling, Eur. Phys. J. C65 (2010) 433.

13. R. Staszewski, P. Lebiedowicz, M. Trzebiński, J. Chwastowski and A. Szczurek, Acta Phys. Polon. B42 (2011) 1861.

14. P. Lebiedowicz and A. Szczurek, Phys. Rev. D83 (2011) 076002.

15. A. Breakstone et al. [ABCDHW Collaboration], Z. Phys. C42 (1989) 387.

16. A. Breakstone et al. [ABCDHW Collaboration], Z. Phys. C48 (1990) 569.

17. M. Glück, D. Jimenez-Delgado, E. Reya and C. Schuck, Phys. Lett. B664 (2008) 133.

18. M. Glück, E. Reya and A. Vogt, Z. Phys. C67 (1995) 433.

19. O. Nachtmann, talk "A model for high-energy soft reactions" at ECT* workshop on Exclusive and diffractive processes in high energy proton-proton and nucleus-nucleus collisions, Trento, February 27 - March 2, 2012.

20. A. Szczurek and J. Speth, Nucl. Phys. A 728 (2003) 182. 